\title{
Prevalence of Malaria during Pregnancy and Antimalarial Intervention in an Urban Secondary Health Care Facility in Southern Nigeria
}

\author{
Ehijie F.O. Enato ${ }^{a}$ Augustine O. Okhamafe ${ }^{b}$ Eugene E. Okpere ${ }^{c}$ \\ Frederick I. Oseji $^{\text {d }}$ \\ Departments of a Clinical Pharmacy and Pharmacy Practice and ${ }^{b}$ Pharmaceutics and Pharmaceutical Technology, \\ Faculty of Pharmacy, and ' Department of Obstetrics and Gynaecology, Faculty of Medicine, University of Benin, \\ and ${ }^{d}$ St. Philomena Catholic Hospital, Benin City, Nigeria
}

\section{Key Words}

Antimalarial intervention, urban secondary health care facility $\cdot$ Pregnancy, malaria prevalence $\cdot$ Prevalence, malaria

\begin{abstract}
Objective: To investigate the prevalence of malaria during pregnancy and antimalarial interventions in an urban secondary health care facility. Subjects and Methods: Of 432 pregnant women who delivered or were attending an antenatal clinic in a secondary health care facility in Benin City, Nigeria, 199 were recruited for the study. Demographic data were obtained from the pregnant women at delivery, and maternal peripheral, placental, and cord blood samples were collected for microscopy. Among the antenatal clinic attendees, a questionnaire was used to assess their antimalarial preventive measures. Results: Of the 199 pregnant women, 60 (30\%) reported that they had had malaria during the preceding 3 months, and a majority of them (85\%) used chloroquine. Almost all reported the drug was efficacious $(98 \%)$ and well tolerated (80\%). Only $18(13 \%), 14(10 \%)$, and 2 (1\%) of the women had positive maternal peripheral, placental, and cord blood parasitaemia, respectively. The geometric mean $( \pm S D)$ numbers of malaria parasites per microliter were $636.06 \pm 1,450.11$ in peripheral blood, 4,250.36 \pm
\end{abstract}

$13,866.01$ in placental blood, and $59.50 \pm 27.58$ in umbilical cord blood. Only 31 (12\%) and $13(5 \%)$ of antenatal women believed in the efficacy of insecticide-treated bed nets or sulphadoxine/pyrimethamine-based intermittent preventive therapy as antimalarial preventive measures, respectively, while $23(9 \%)$ and 31 (12\%), respectively, reported they currently use them. Conclusions: Despite the common occurrence of malaria during pregnancy, there was a limited knowledge and use of the recommended antimalarial interventions by pregnant women attending the antenatal clinic of this health care facility. There is, therefore, an urgent need to increase the implementation of antimalarial interventions during pregnancy.

Copyright $\odot 2007$ S. Karger AG, Basel

\section{Introduction}

Malaria is a major threat to public health and socioeconomic development in sub-Saharan Africa. Pregnant women and children are most affected, resulting in significant maternal, perinatal and fetal morbidity and mortality. About one to three million children die each year, while about 24 million pregnancies are threatened in Africa as a result of malaria $[1,2]$. The impact of malaria in pregnancy is most dramatic in populations, where preg-

\section{KARGER \\ Fax +41613061234 \\ E-Mail karger@karger.ch}

www.karger.com
(C) 2007 S. Karger AG, Basel

1011-7571/07/0163-0240\$23.50/0

Accessible online at:

www.karger.com/mpp
Dr. Ehijie F.O. Enato

Department of Clinical Pharmacy and Pharmacy Practice

Faculty of Pharmacy, University of Benin

Benin City 300001 (Nigeria)

Tel.+234802359 7448, E-Mail enatoefo@uniben.edu or ehise2002@yahoo.com 
Table 1. Demographic, clinical, and obstetric characteristics of the women at delivery

\begin{tabular}{lc}
\hline Characteristic & Values $(95 \% \mathrm{CI})$ \\
\hline Mean age, years & $29(28.31-29.69)$ \\
At least secondary school education, \% & $90(86-94)$ \\
Mean body weight, kg & $72.59(70.94-74.24)$ \\
Mean gestational week at delivery & $36.42(36.20-36.64)$ \\
Mean gravidity & $3.13(2.90-3.36)$ \\
Mean parity & $2.03(1.82-2.24)$ \\
Mean axillary temperature, ${ }^{\circ} \mathrm{C}$ & $36.72(36.64-36.80)$ \\
Geometric mean peripheral parasitaemia/ $\mu \mathrm{l}(\mathrm{n}=18)$ & $636.06(377.42-894.70)$ \\
Geometric mean placental parasitaemia/ $\mu \mathrm{l}(\mathrm{n}=14)$ & $4,250.36(1,718.61-6,782.11)$ \\
Geometric mean umbilical cord parasitaemia/ $\mu \mathrm{l}(\mathrm{n}=2)$ & $59.50(29.87-89.13)$ \\
Anaemia (packed cell volume $<30 \%), \%$ & $17(12-22)$ \\
Mean singleton birth weight, kg $\%$ & $3.21(3.14-3.28)$ \\
Reported malaria within 3 months prior to enrollment, \% & $30(24-36)$ \\
Reported chloroquine use within 3 months prior to & $85(81-90)$ \\
$\quad$ enrollment, $\%$ &
\end{tabular}

nant women have little or no pre-existing immunity. However, in areas of high and stable malaria transmission, which predominate in sub-Saharan Africa, the effects of ill health are particularly apparent in first and second malaria-exposed pregnancies. In multigravidae, maternal morbidity due solely to malaria is uncommon, and the major detrimental effects of the infection are low birth weight and maternal anaemia [3,4]. The overwhelming socio-economic and developmental burden of malaria in endemic regions has resulted in increased global attention [5]. The objective of this study was to investigate the prevalence of malaria during pregnancy and antimalarial interventions.

\section{Subjects and Methods}

The study was carried out at St. Philomena Catholic Hospital, a 160-bed secondary health care facility in Benin City, Nigeria. Of 432 pregnant women who delivered at the hospital during the periods of July to November 2004 and January to July 2005, 199 were recruited for the study after verbal consent had been obtained. The remaining 233 women were excluded because of delivery by caesarean section $(\mathrm{n}=197)$, stillbirth $(\mathrm{n}=34)$, and twin delivery $(n=2)$. Of the 199 study participants, thick-film slides of blood obtained from maternal peripheral, placental, and cord were examined for 140 women [6]. In the remaining 59 cases, the slides were discarded because it was observed that the laboratory assistant did not filter the Giemsa solution prior to staining, thus resulting in a high number of artefacts. However, these 59 women did not differ in demographic and obstetric profiles from those whose slides were examined. In addition, a self-administered questionnaire was used to assess the antimalarial preventive measures of a sample of 250 pregnant women attending the antenatal clinic of the same hospital during the same study periods.

Antimalarial Intervention in Southern Nigeria

\section{Laboratory Investigation}

Malaria parasites were counted microscopically on Giemsastained thick-blood films per 100 high-power fields. The parasites were counted against 200 white blood cells (WBCs) in the same fields. Parasite densities were calculated on the basis of assumed WBCs of $8,000 / \mu \mathrm{l}$ blood. Total WBCs were not measured to correct the assumed parasite densities. A smear was considered negative, if no parasites were seen in 200 oil immersion fields in a thick-blood film. The haematocrit was determined using a microhaematocrit centrifuge; the birth weight was determined using a tray scale. The gestational age was determined using fundal height and fetal size. Ethical approval for the study was obtained from the Ethical Review Committee, University of Benin Teaching Hospital, Benin City, Nigeria.

\section{Data Analysis}

Data were entered into Microsoft Spreadsheet and sorted. Thereafter, the data were analyzed by calculating percentage frequencies, mean values, standard deviation, and $95 \%$ confidence interval, where applicable. Differences between mean values were analyzed using GraphPad Instat ${ }^{\circledR}$ version 2.05a.

\section{Results}

The demographic characteristics of the women at delivery are shown in table 1 . Obstetric history showed that the gestational age at delivery ranged from 25 to 41 weeks, and only $19 \%$ of the study participants were primigravidae. Of the 199 pregnant women, 148 (74\%) had preterm delivery. Of the 148 who had preterm delivery, 139 (94\%), delivered at 36 weeks of gestation. Of the 199 live births, $12(6 \%)$ had a low birth weight. Of the 60 women $(30 \%)$ who reported to have had malaria during the 3-month recall period, chloroquine was most commonly used in $51(85 \%)$ for the management of the condition. Forty 
Fig. 1. Perception of efficacy and practice of antimalarial preventive measures by pregnant women attending an antenatal clinic in Nigeria $(\%, n=250)$. The design allowed for weighted responses. ITN = Insecticide-treated bed net; IPT = intermittent preventive therapy.



women (78\%) reported that the chloroquine was prescribed by a doctor. When further questioned on the effectiveness of the drug after therapy, 43 women (98\%) of those who responded reported the drug was effective. The adverse effects reported after chloroquine therapy were itching in 10 women (20\%), dizziness in 4 patients $(8 \%)$, and vomiting in 2 women (4\%), while the rest reported none or could not recall any adverse effect.

Maternal peripheral, placental, and cord blood parasitaemias were found in 18 (13\%), 14 (10\%), and 2 (1\%) patients, respectively. Mean $( \pm S D)$ packed cell volume, birth weight, and body temperature of the women with malaria were $32.22 \pm 4.26 \%, 2.97 \pm 0.43 \mathrm{~kg}$, and $36.57 \pm 0.43^{\circ} \mathrm{C}$, respectively, while the values of the women without malaria were $33.43 \pm 4.12 \%, 3.18 \pm 0.54 \mathrm{~kg}$, and $36.70 \pm$ $0.56^{\circ} \mathrm{C}$, respectively. There were no significant differences in these parameters in women with or without malaria $(p>0.05)$. The perception of the efficacy and practice of antimalarial interventions by antenatal clinic attendees is shown in figure 1 . Only 91 (36\%) and 37 (15\%) of the patients reported chloroquine or sulphadoxine/pyrimethamine, respectively, was safe during pregnancy.

\section{Discussion}

The data from this study indicate that malaria is a common health care problem among pregnant women attending the antenatal clinic in this health care facility.
Malaria during pregnancy may have contributed to the observed negative pregnancy outcomes, as previously reported by Ibhanesebhor and Okolo [7]. Chloroquine was most commonly used in the management of malaria in pregnancy, since a previous national antimalarial treatment guideline recommended chloroquine as the firstline drug in the management of uncomplicated malaria in pregnancy [8]. However, due to perceived high prevalence of Plasmodium falciparum strains resistant to the drug, the guideline was revised and oral quinine recommended [9]. Though a majority reported that chloroquine was effective for them after therapy, further clinical studies are required for verification.

Despite receiving health education at the antenatal clinic, the respondents had a limited knowledge and a low utilization of the recommended antimalarial interventions. This study took place in a hospital in an urban setting; however, the situation may be worse among pregnant women in general, especially in peripheral health care facilities. Therefore, efforts to address these findings are recommended in order to ensure that global malaria targets are attained in the study area. Although this study has presented some important findings, the possibility of selection bias cannot be ruled out, considering the demographic and clinical profile of the study participants. The sample size is small, and this may affect the confidence interval. Furthermore, the method used in the diagnosis of placental malaria was not very sensitive. Nevertheless, our findings have provided some evidence of the need to 
increase antimalarial intervention for pregnant women in Nigeria, as these findings may reflect the situation in many parts of the country.

\section{Conclusions}

Despite the common occurrence of malaria during pregnancy, knowledge and use of recommended antimalarial interventions by pregnant women attending the antenatal clinic of this health care facility were found to be very limited. There is, therefore, an urgent need to expand the implementation of antimalarial interventions during pregnancy. Future studies should consider using a histopathological method in the diagnosis of placental malaria.

\section{Acknowledgment}

We are grateful to Bernd Robioneck for his critical review of and comments on the manuscript.

\section{References}

1 Ntoumi F, Djimde AA, Mbacham W, Egwang $\mathrm{T}$ : The importance and future of malaria research in Africa. Am J Trop Med Hyg 2004; 71(2 Suppl):IV.

2 Leke R: Intermittent preventive treatment of malaria in pregnancy (Cameroon experience). Acta Trop 2005;95(Suppl):S2-S3.

3 van Geertruyden J, Thomas F, Erhart A, D'Allessandro U: The contribution of malaria in pregnancy to perinatal mortality. Am J Trop Med Hyg 2004;71(2 Suppl):35-40.
-4 Steketee RW, Nahlen BL, Parise M, Menendez C: The burden of malaria in pregnancy in malaria-endemic areas. Am J Trop Med Hyg 2001;64(1-2 Suppl):28-35.

5 Global Strategic Plan: Roll Back Malaria 2005-2015. Roll Back Malaria Partnership. Geneva, World Health Organization, 2005.

6 Rogerson SJ, Mkundika P, Kanjala MK: Diagnosis of Plasmodium falciparum malaria at delivery: comparison of blood film preparation methods and of blood films with histology. J Clin Microbiol 2003;41:1370-1374.
7 Ibhanesebhor SE, Okolo AA: Placental malaria and pregnancy outcome. Int J Gynaecol Obstet 1992;37:247-252.

8 Federal Ministry of Health: National Malaria Control Policy for Nigeria. Yaba, Lagos, National Malaria and Vector Control Division, 2001.

9 Federal Ministry of Health: National Malaria Control Policy for Nigeria. Yaba, Lagos, National Malaria and Vector Control Division, 2004. 